\title{
Impacto de la vacuna conjugada en la incidencia de meningitis por Haemophilus influenzae en el Distrito Federal de Brasil: resultados de tres años de seguimiento
}

\author{
Helen Selma de Abreu Freitas ${ }^{1}$ y Edgar Merchán-Hamann ${ }^{2}$
}

Forma de citar

Freitas HSA, Merchán-Hamann E. Impacto de la vacuna conjugada en la incidencia de meningitis por Haemophilus influenzae en el Distrito Federal de Brasil: resultados de tres años de seguimiento. Rev Panam Salud Publica. 2006;19(1):33-7.

RESUMEN Introducción. Haemophilus influenzae del serotipo b (Hib) es todavía un importante agente causal de procesos infecciosos. Su variante encapsulada es la causa de formas invasoras de enfermedad. En algunas poblaciones aborígenes, la incidencia de enfermedades causadas por Hib es mayor de 400 por 100000 niños menores de 5 años. En los decenios de 1970 y 1980, tras la identificación de anticuerpos protectores contra la cápsula de Hib, se desarrollaron vacunas contra este microorganismo.

Objetivo. Estimar el impacto que ha tenido desde su introducción, en marzo de 1998, la vacunación contra Hib en el Distrito Federal de Brasil.

Método. Con los datos de base poblacional del Sistema de Vigilancia de la Secretaría de Salud del Distrito Federal de Brasil, se compararon las tasas de incidencia de meningitis correspondientes a los tres años anteriores y posteriores a la introducción de la vacuna. Se compararon también los cambios en su tendencia.

Resultados. Al comparar los dos períodos se advierte una reducción de aproximadamente $90 \%$ en la incidencia de meningitis por Hib, cambio que no ocurre en el caso de las meningitis ocasionadas por otros agentes bacterianos. Se registró un aumento proporcional de los casos entre los niños de 6 meses de edad y menores, debido a la reducción de la incidencia entre los mayores de esa edad.

Conclusión. La introducción de la vacuna conjugada en el Distrito Federal de Brasil redujo de 168 por 100000 (1995-1997) a 15 por 100000 (1999-2001) la incidencia de meningitis por Hib entre niños de 7 a 35 meses. Esto representa una reducción de 91,1\%.

Palabras clave Meningitis; Haemophilus influenzae; vacunas contra Haemophilus.

Coordinación General de Enfermedades Transmisibles, Secretaría de Vigilancia en Salud, Ministerio de Salud del Brasil. Toda correspondencia deberá dirigirse a: Helen Selma de Abreu Freitas, NESP, CLN 406, Bloco A, Sala 211, Brasilia DF, CEP 70.847-510, Brasil. Teléfono: 55-61-3340-6863. Correo electrónico: helen.freitas@saude.gov.br

2 Núcleo de Estudios de Salud Pública (NESP), Centro de Estudios Avanzados Multidisciplinarios (CEAM), Universidad de Brasilia (UnB), Brasilia DF, Brasil.
Haemophilus influenzae del serotipo b (Hib), un bacilo gramnegativo, es todavía causa importante de procesos infecciosos. La variante encapsulada de Hib es el principal agente causal de las formas invasoras de las enfermedades ocasionadas por esta especie, en particular otitis media, osteomielitis, neumonía y meningitis $(1,2)$. Ciertamente, su frecuente presencia en la bucofaringe en la población en general ha favorecido el contagio a edades tempranas. Por tal motivo, casi todas las formas de meningitis producidas por este agente se observan antes de los 5 años de edad; de hecho, se han regis- 
trado incidencias anuales de hasta 400 casos por 100000 niños menores de 5 años en algunas poblaciones aborígenes de Estados Unidos y Australia, y cercanas a 35 casos por 100000 entre indígenas de América Latina (3-7).

Haemophilus influenzae del serotipo b (Hib) posee una cápsula de polisacárido polirribosil-ribitolfosfato (PRP) que determina la virulencia de esta variedad $(8,9)$. La identificación de anticuerpos protectores contra esa cápsula celular posibilitó la elaboración de una vacuna contra Hib (10, 11, 12). En el decenio de 1970 se sometieron a prueba las primeras vacunas contra Hib elaboradas con el polisacárido capsular PRP, las cuales mostraron baja eficacia en niños menores de 2 años (13-15). A partir de 1985 se probaron vacunas producidas mediante conjugación de la cápsula de PRP con una proteína transportadora. En la primera se conjugaba el PRP con el toxoide diftérico para producir PRP-D. Más tarde se crearon otras vacunas mediante la conjugación de la proteína capsular de Hib con la membrana externa (OMP, outer membrane protein) de Neisseria meningitidis del grupo C, complejo que se designó PRP-OMP, o con el toxoide tetánico, para obtener la vacuna PRP-T (12,16-18). En todas las regiones donde se implantó el uso universal de la vacuna se observó un descenso importante de la incidencia de enfermedades invasoras causadas por Hib (19-21, 22-25), si bien estudios posteriores acerca de la eficacia de las vacunas conjugadas señalaron resultados distintos en algunas poblaciones $(26,27)$.

Entre los cuadros clínicos que produce Haemophilus influenzae, el que con mayor frecuencia se diagnostica y es objeto de identificación etiológica es la meningitis, cuya gravedad y criterios de diagnóstico establecen las condiciones para un mejor conocimiento de los casos. Ocurre así particularmente en los países en desarrollo, donde es escasa la información sobre otros procesos infecciosos causados por especies de Haemophilus $(4,28,29)$. El conocimiento de los casos de meningitis por Haemophilus influenzae ha proporcionado información sobre las característi- cas epidemiológicas de la enfermedad, además de brindar parámetros para el establecimiento de medidas de control y la evaluación de sus resultados.

\section{ANTECEDENTES}

Para 1998, año en que se introdujo la vacuna, la población estimada del Distrito Federal (DF) de Brasil era de 1923404 habitantes e incluía un número estimado de 190963 niños menores de 5 años. La proporción de niños menores de esta edad era de $11 \%$, o cerca de $50 \%$ de la estimada para los países en desarrollo $(4,28)$.

Los servicios de vigilancia epidemiológica de cada hospital notifican diariamente los casos atendidos de meningitis a la Secretaría de Salud del Distrito Federal (Secretaria de Saúde do Distrito Federal, SES/DF), la cual concentra la información procedente de las unidades asistenciales de todo el DF. Para tal efecto se llena un formulario, estandarizado por el Ministerio de Salud (Ministério da Saúde, MS), que contiene datos clínicos, de laboratorio y de atención clínica pertinentes al caso. El respaldo de laboratorio está constituido por los laboratorios de cada hospital y un laboratorio central de salud pública (ISDF/LACEN), en el cual se procesan más de $90 \%$ de las muestras de líquido cefalorraquídeo (LCR) obtenidas en el DF.

En el DF, la cobertura vacunal se mantiene en un nivel aproximado de $95 \%$, según encuestas epidemiológicas realizadas a partir de 1986 (30).

Entre 1995 y 1997 se registró un promedio de 381 casos de meningitis cada año en personas de todas las edades residentes en el DF. Las tasas de incidencia de meningitis bacteriana por 100000 habitantes menores de 5 años fueron de 117,4, 122,4 y 112,0 en los años de 1995, 1996 y 1997, respectivamente. Entre los menores de 1 año, las tasas de incidencia de meningitis por Haemophilus influenzae fueron 111,7, 106,3 y 83,2, respectivamente, en los tres años que precedieron a la introducción de la vacuna, es decir 1995, 1996 y 1997. Entre los menores de 5 años, las cifras correspondientes a estos años fueron 43,4, 33,9 y 27,9, respectivamente. De las muestras de LCR obtenidas de pacientes con sospecha clínica de meningitis y remitidas a examen, $12,5 \%$ resultaron positivas en cuanto a la presencia de bacterias. Entre las meningitis bacterianas de causa confirmada, Haemophilus influenzae fue el agente causal de 25,7\% de los casos en el período de 1995 a 1997. Del total de cepas aisladas, $97 \%$ correspondieron al serogrupo b, $2 \%$ resultaron inclasificables y solo $1 \%$ no se examinaron.

Los servicios de salud del Distrito Federal emprendieron el programa de vacunación contra Hib en abril de 1998, cuando se administraron las primeras dosis en niños, sin indicación especial. La vacuna elegida fue la PRP-T. Para ese año, la campaña consistió en la vacunación de todos los niños de hasta 1 año de edad, conforme a esquemas apropiados para cada grupo etario: de 2 a 6 meses de edad, tres dosis a intervalos de 2 meses; de 7 a 11 meses de edad, dos dosis a un intervalo de 2 meses. A partir de 1999 se siguió el calendario nacional: tres dosis, administradas a los 2, 4 y 6 meses de edad, sin refuerzos. La cobertura de la vacuna conjugada fue de $100 \%$ desde su introducción en el Distrito Federal.

\section{MÉTODOS}

Con el apoyo de datos de base poblacional, se evaluó el impacto de la introducción de la vacuna conjugada (PRP-T) en la reducción del número de casos de meningitis por Hib en el DF a partir de 1998.

La principal fuente de información fue el Programa de Control de Meningitis de la SES/DF, que recibe y concentra los datos del Distrito Federal de Brasil. Se consideraron "casos de meningitis por Haemophilus influenzae" los pacientes que presentaron un cuadro clínico y citoquímico de LCR compatible con meningitis, que tuvieron aislamiento de este agente por cultivo del LCR o su identificación por medio de pruebas de contrainmunoelectroforesis (CIEF) o aglutinación de látex en el LCR. El conjunto de las "otras causas 
bacterianas" (OCB) se definió como todos aquellos casos de meningitis bacteriana en que se identificara el agente causal, excepto Hib y meningococos.

Se estudió el número de casos en los períodos anterior y posterior a la introducción de la vacuna conjugada. Para estimar la eficacia de esta última se compararon las cifras de incidencia de meningitis por Hib anteriores y posteriores a la introducción de la vacuna contra Hib, mediante la ecuación propuesta por Orenstein et al. $(31,32)$ para la evaluación de la eficacia:

$$
\mathrm{VE}=\frac{\mathrm{ARU}-\mathrm{ARV}}{\mathrm{ARU}} \times 100
$$

siendo VE la eficacia vacunal; ARU la tasa de ataque en no vacunados; y ARV la tasa de ataque en vacunados.

Se compararon las incidencias en niños de 7 a 35 meses, grupo que supuestamente estaría inmunizado por haber recibido tres dosis de vacuna. Los períodos comparados fueron 19951997 y 1999-2001. Para calcular la incidencia se adoptaron las cifras de población del grupo etario en estudio, de los años 1996 y 2000, que corresponden a la mitad de cada período analizado y que coinciden con el censo de población.

Para evaluar la posibilidad de que otros factores contribuyeran a reducir el número de casos de meningitis por Hib se compararon las variaciones en las tasas de incidencia de meningitis causada por Hib y las de meningitis por OCB en los trienios anterior (19951997) y posterior (1999-2001) al año de introducción de la vacuna. Con ese fin se calculó la razón de las tasas de incidencia entre las situaciones previa y posterior a la introducción de la vacuna conjugada, tanto para los casos causados por Hib como para los resultantes de OCB. Para calcular las tasas se utilizaron las cifras de población de los años 1996 y 2000, dentro del grupo etario en estudio.

Se describieron los porcentajes acumulados de casos por edad en los períodos anterior y posterior a la introducción de la vacuna conjugada.

\section{RESULTADOS}

Como se aprecia en la figura 1, el número de casos de meningitis por Hib entre menores de 1 año y menores de 5 años en el DF mostró un evidente descenso después de la introducción de la vacuna. Las tasas de incidencia de meningitis por Hib en los trienios que precedieron y sucedieron respectivamente al año de introducción de la vacuna conjugada, 1995-1997 y 1999-2001, fueron de 168 por 100000 y 15 en 100000 entre los niños de 7 a 35 meses, lo cual representa una reducción de 91,1\% cuando se aplica la fórmula de Orenstein mencionada antes. El cuadro 1 muestra la condición vacunal de los niños que tuvieron meningitis por Hib entre 1999 y 2001. Solo en un caso, un niño con vacunación completa tuvo meningitis por Hib.

Entre los niños de 7 a 35 meses la incidencia de meningitis causada por OCB se mantuvo constante en los períodos estudiados (1995-1997 y 19992001), a diferencia de los casos comprobados de meningitis por Hib, que se redujeron súbitamente. En el período de 1995-1997, el número de casos de meningitis por OCB fue de 38 ,

FIGURA 1. Número de casos de meningitis por Haemophilus influenzae en menores de 1 año $(<1 \mathrm{~A})$ y menores de 5 años $(<5 \mathrm{~A})$, por año y trimestre de aparición: Brasilia, DF, 1994-2001

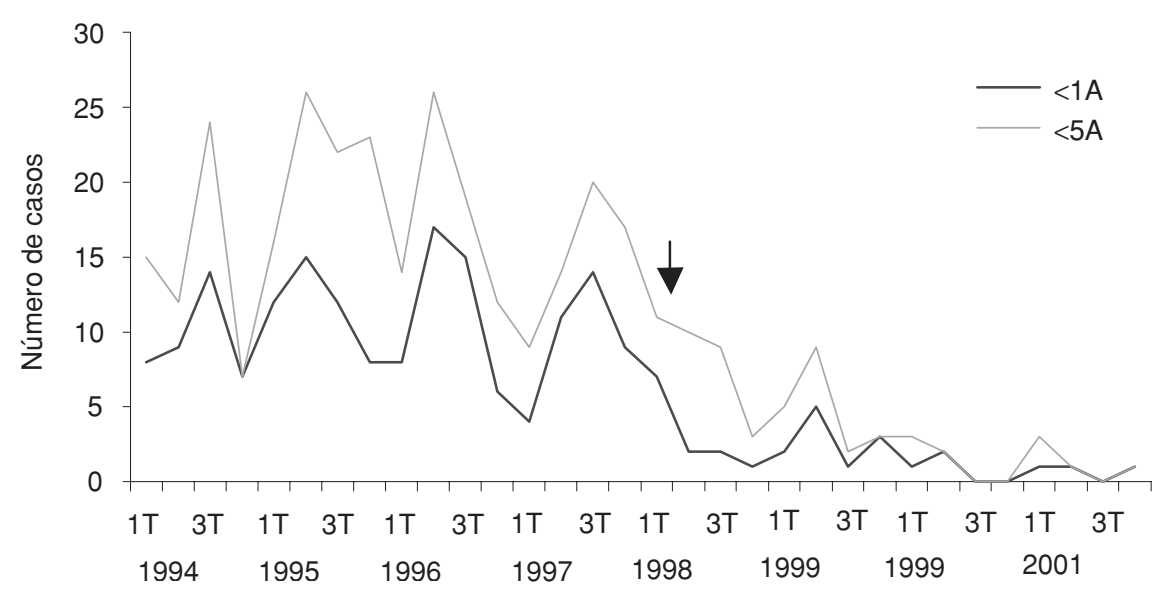

CUADRO 1. Pacientes de 7 a 35 meses de edad, ${ }^{\text {a }}$ residentes del Distrito Federal de Brasil, que tuvieron meningitis por Haemophilus influenzae entre 1999 y 2001

\begin{tabular}{lcccl}
\hline Caso & $\begin{array}{c}\text { Edad } \\
\text { (meses) }\end{array}$ & $\begin{array}{c}\text { Fecha de la } \\
\text { enfermedad }\end{array}$ & Serotipo & Situación vacunal \\
\hline DAAA & 12 & $07 / 04 / 1999$ & $\mathrm{~b}$ & Se desconoce \\
RNS & 7 & $20 / 04 / 1999$ & $\mathrm{~b}$ & No se vacunó \\
SSO & 7 & $04 / 06 / 1999$ & $\mathrm{~b}$ & No se vacunó \\
WOSF & 8 & $25 / 08 / 1999$ & $\mathrm{~b}$ & Se desconoce \\
NEF & 7 & $17 / 10 / 1999$ & $\mathrm{~b}$ & Se vacunó (dos dosis) \\
LCR & 7 & $04 / 11 / 1999$ & $\mathrm{~b}$ & Se vacunó (una dosis) \\
LCO & 24 & $21 / 02 / 2000$ & $\mathrm{~b}$ & Se desconoce \\
MOP & 35 & $06 / 03 / 2000$ & $\mathrm{~b}$ & No se vacunó \\
NAM & 12 & $12 / 03 / 2001$ & $\mathrm{~b}$ & Se vacunó (tres dosis) \\
NGD & 8 & $30 / 10 / 2001$ & $\mathrm{a}$ & Se vacunó (tres dosis)
\end{tabular}

Fuente: Base de Datos de Meningitis, Departamento de Saúde Pública, Secretaria de Saúde do Distrito Federal, Brasil. a Para el año de 1999 se presentan los casos de los grupos de 7 a 23 meses, que son las edades recomendadas para la vacunación. 
mientras que el de meningitis por Hib fue de 153. Tras la introducción de la vacuna, en el período 1999-2001 se registraron 42 casos de meningitis por OCB y solo 15 por Hib. La razón de incidencias anterior y posterior a la introducción de la vacuna conjugada (incidencia anterior/incidencia posterior) fue de 11,2 para Hib y de 1,05 para OCB, lo que constituye una reducción 11 veces mayor de los casos de meningitis causados por Hib que de los causados por OCB. La comparación de las tasas de incidencia de enfermedades causadas por estos agentes en los períodos señalados respalda la conclusión de que la introdución de la vacuna conjugada fue el factor que determinó el rápido descenso de la incidencia.

Se comprobó un aumento proporcional de los casos entre los niños menores de 7 meses de edad, así como entre los mayores de 2 años, lo cual es un efecto esperado del cambio producido por la vacuna. Los casos verificados en niños menores de 7 meses representaron $24 \%$ del total diagnosticado. Tras la introducción de la vacuna la proporción se elevó a 35\%.

\section{DISCUSIÓN Y CONCLUSIÓN}

La frecuencia de meningitis por Hib verificada en el Distrito Federal de Brasil en el período previo a la introducción de la vacuna contra $\mathrm{Hib}$ correspondió a las estimaciones que registran las fuentes bibliográficas (3).

La comparación entre la incidencia de meningitis por Hib anterior y posterior a la introducción de la vacunación en pacientes del mismo grupo de edad demuestra una reducción mayor de $90 \%$ en el número de casos, lo cual puede atribuirse directamente a la vacuna conjugada, ya que esta variación incide solamente en los casos de meningitis causadas por Hib. La reducción lograda del número de casos apunta a una efectividad y eficacia semejantes a las señaladas en estudios de otros países $(23,24,26)$.

El aumento proporcional de los casos que se producen durante los 6 primeros meses de edad indica que Haemophilus influenzae del serotipo $b$ sigue siendo un agente patógeno importante en ese período inicial de la vida. Conviene recalcar que la meningitis es solamente una de las entidades clínicas causadas por Hib, a lo cual se añade el hecho de que la vacunación de las embarazadas protege a los recién nacidos $(2,33,34)$, pudiendo ser en el futuro un nuevo recurso para la protección de los niños de este grupo de edad.

\section{REFERENCIAS}

1. Focaccia R. Meningites bacterianas. En: Veronesi R, Focaccia R. Tratado de infectologia. São Paulo: Ed. Atheneu; 2002;827-41.

2. Benenson AS (ed.). Manual para el control de las enfermedades transmisibles, $17^{\mathrm{a}} \mathrm{ed}$. Washington, D.C.: Organización Panamericana de la Salud; 1997. (Publicación Científica No. 564.)

3. Peltola H. Haemophilus influenzae type b disease and vaccination in Latin America and the Caribbean. Pediatr Infect Dis J. 1997;16(8): 780-7.

4. Steinhoff MC, Santosham M. The use of $H$. influenzae type $\mathrm{b}$ vaccines in the WHO expanded program of immunization: a preliminary feasibility assessment. USAID and WHO/EPI Division. Baltimore: School of Hygiene and Public Health, Johns Hopkins University; 1993. (Scientific Monograph.)

5. Bijlmer HA. World-wide epidemiology of Haemophilus influenzae meningitis: industrialized versus non-industrialized countries. Vaccine. 1991; 9(suppl.):S5-8.

6. Dajani AS, Asmar BI, Thirumoorthi MC. Systemic Haemophilus influenzae disease: an overview. J Pediatrics. 1979:94(3):355-64.

7. Smith EW, Haynes RE. Changing incidence of Hemophilus influenzae meningitis. Pediatrics. 1972; 50(5):723-7.

8. Fothergill LD, Wright J. Influenzal meningitis: the relation of age incidence to the bactericidal power of blood against the casual organism. J Immunol. 1933;24:273-84.

9. Alexander HE, Heidelberger M, Leidy G. The protective element in type $\mathrm{b}$ Influenzae rabbit serum. Yale J Biol Med. 1945;16:425-34.
10. Schneerson R, Rodrigues LP, Parke JC, Robbins JB. Immunity to disease caused by Haemophilus influenzae type b. J Immunol. 1971;107(1):397-401.

11. Robbins JB, Parke JC, Scheneeson R, Whisnant JK. Quantitative measurement of "natural" and immunization-induced Haemophilus influenzae type $\mathrm{b}$ capsular polisaccharide antibodies. Pediatr Res. 1973;7(3):103-10.

12. Clements DA, Gilbert GL. Immunisation for the prevention of Haemophilus influenzae type b infections: a review. Aust N Z J Med. 1990; 20(6):828-32.

13. Peltola H, Käyhty H, Sivonen A, Mäkelä HP. Haemophilus influenzae type $\mathrm{b}$ capsular polysaccharide vaccine in children: a double-blind field study of 100000 vaccinees 3 months to 5 years of age in Finland. Pediatrics. 1977; 60(5): 730-7.

14. Shapiro ED, Murphy TV, Wald ER, Brady CA. The protective efficacy of Haemophilus b polysaccharide vaccine. JAMA. 1988;260(10): 1419-22.

15. CDC Immunization Practices Advisory Committee (ACIP). Update: Prevention of Haemophilus influenzae type $\mathrm{b}$ disease. MMWR. 1988; 37(2):13-6.

16. Capeding MRZ, Nohynek H, Pascual L, Sombrero L, Sunico E, Eskola J, et al. Antibody responses to three Haemophilus influenzae type $b$ (Hib) conjugate vaccines in Filipino infants. Am J Trop Med Hyg. 1996;55(5):516-20.

17. Parke JC, Scheerson R, Reimer C, Black C, Welfare $\mathrm{S}$, et al. Clinical and immunologic responses to Haemophilus influenzae type b- tetanus toxoid conjugate vaccine in infants injected at 3, 5, 7 and 18 months of age. J Pediatrics. 1991;118(2):184-90.

18. Eskola J, Käyhty H, Takala AK, Peltola H, Rönnberg PR, et al. A randomized prospective field trial of a conjugate vaccine in the protection of infants and young children against invasive Haemophilus influenzae type $\mathrm{b}$ disease. N Engl J Med. 1990;323(20):1381-7.

19. Peltola H. Haemophilus influenzae type $\mathrm{b}$ disease and vaccination in Europe: lessons learned. Pediatr Infect Dis J. 1998;17(suppl.): S126-32.

20. Peltola H, Kilpi T, Anttila M. Rapid disappearance of Haemophilus influenzae type $b$ meningitis after routine childhood immunization with conjugate vaccines. Lancet 1992; 340(8819):592-4.

21. Alphen LV, Spanjaard L, Ende AV, Schurman I, Dankert J. Effect of nationwide vaccination of 3-month-old infants in the Netherlands with conjugate Haemophilus influenzae type $\mathrm{b}$ vaccine: high efficacy and lack of herd immunity. J Pediatrics. 1997;131(6):869-73.

22. Landaverde M, DiFabio JL, Ruocco IL, Quadros C. Introducción de la vacuna conjugada contra Haemophilus influenzae tipo b en Chile y Uruguay. Rev Panam Salud Publica. 1999;10(3):200-6.

23. Slack MPE, Azzioardi HJ, Hargreves RM, Ramsay ME. Enhanced surveillance of invasive Haemophilus influenzae disease in England, 1990 to 1996: impact of conjugate vaccines. Pediatr Infect Dis J. 1998;17(suppl. 9): S204-7. 
24. Dinckinson F, Pérez AE, Galindo MA, Quintana I. Impacto de la vacunación contra Haemophilus influenzae tipo b en Cuba. Rev Panam Salud Publica. 2001;10(3):169-73.

25. Murphy TV, White KE, Pastor P, Gabriel L, Medley F, Granoff DM, et al. Declining incidence of Haemophilus influenzae type $\mathrm{b}$ disease since introduction of vaccination. JAMA. 1993;269(2):246-8.

26. Campbell H, Byass P, Ahonkhai VI, Vella PP, Grenwood BM. Serologic responses to an Haemophilus influenzae type $\mathrm{b}$ polyssaccharideNeisseria meningitidis outer membrane protein conjugate vaccine in very young Gambian infants. Pediatrics. 1990; 86(1):102-7.

27. Mulholland EK, Byass P, Campbell H, Fritzell B, Greenwood AM, Todd J, et al. The immunogenicity and safety of Haemophilus influenzae type b-tetanus toxoid conjugate vaccine in Gambian infants. Ann Trop Paediatr. 1994;14(3):183-8.
28. Levine OS, Schwartz B. The rationale for population based surveillance for Haemophilus influenzae type $b$ meningitis. Pediatr Infect Dis J. 1998;17(suppl. 9);S195-8.

29. Biljmer HA, Alphen LV. A prospective, population-based study of Haemophilus influenzae type $b$ meningitis in the Gambia and the possible consequences. J Infect Dis. 1992; 165(suppl. 1):S29-32.

30. Carvalho LF. Proposta de Estratégia da Vacinação contra o Sarampo no DF; Programa de Imunização do Distrito Federal. Brasília: Departamento de Saúde Pública, Secretaria de Saúde do Distrito Federal; 1995.

31. Orenstein WA, Berner RH, Hinan AR. Assessing vaccine efficacy in the field. Epidemiol Rev. 1988;10:212-39.

32. Halloran ME, Haber M, Longini IM, Struchiner CJ. Direct and indirect effects in vaccine efficacy and effectiveness. Am J Epidemiol. 1991;133(4):323-30.
33. Santosham M, Englund JA, Mcinnes P, Croll J, Thompson CM, et al. Safety and antibody persistence following Haemophilus influenzae type $\mathrm{b}$ conjugate or pneumococcal polysaccharide vaccines given before pregnancy in women of childbearing age and their infants. Pediatr Infect Dis J. 2001;20(10):931-40.

34. Englund JA, Glezen WP. Maternal immunization with Haemophilus influenzae type $\mathrm{b}$ vaccines in different populations. Vaccine. 2003; 21(24):3455-9.

Manuscrito recibido el 17 de agosto de 2004. Aceptado para publicación, tras revisión, el 18 de agosto de 2005.

ABSTRACT Introduction. Type b Haemophilus influenzae (Hib) continues to be an important causative agent of various infectious processes, and its encapsulated strains cause invasive disease. In some aboriginal populations, the incidence of Hib infections in children under five is greater than 400 per 100 000. In the seventies and eighties, vaccines against Hib were developed after antibodies against the capsular component were identified. The objective of this paper was to estimate the impact that the vaccine against Hib has had in Brazil's Federal District since it was introduced in March of 1998. Methods. Using population-based data obtained from Brazil's Federal District's Ministry of Health's Surveillance System, rates of incidence of meningitis during the three years that preceded and that followed the introduction of the anti-Hib vaccine were compared. Comparisons were also drawn between changes in the trends observed. Results. A comparison of the data from the two periods showed a decrease of approximately $90 \%$ in the incidence of Hib meningitis, but no concomitant decrease in other forms of bacterial meningitis was noted. There was also a proportional increase in the number of cases among infants 6 months of age and under due to a drop in the incidence of the disease in children older than 6 months.

Conclusion. As a result of the introduction of the conjugated anti-Hib vaccine in Brazil's Federal District, the incidence of Hib meningitis among children 7 months to 35 months of age dropped from 168 per 100000 to 15 per 100000 (91.1\%).

Key words Meningitis, Haemophilus; Haemophilus; Haemophilus infections; bacterial vaccines. 